\title{
BULYGIN-REDONDO: DESENCUENTROS CERCANOS SOBRE ENUNCIADOS HARTIANOS DEL TERCER TIPO
}

\author{
María Gabriela Scataglini \\ Universidad de Buenos Aires \\ g_scataglini@hotmail.com
}

RESUMEN: En este texto comparo las concepciones de Eugenio Bulygin y Cristina Redondo acerca de aquellos enunciados que dan cuenta de que en una sociedad se aceptan ciertas reglas como pautas de conducta. Me concentro en la pregunta acerca de qué es lo que posibilita al observador "identificar", "reconocer" normas jurídicas válidas en cuanto tales. Inspirada en el enfoque wittgensteiniano acerca del seguimiento de reglas — retomado a su vez por la crítica de Brandom al "regularismo"-, planteo una respuesta que discrepa de la de Bulygin y coincide con la de Redondo en un punto en el que ambos difieren; mas señalo una sutil - aunque crucialdiferencia con esta última.

PALABRAS CLAVE: punto de vista interno, descriptivo/prescriptivo, seguimiento de reglas, observador, "regularismo"

SUMMARY: In this text I compare Eugenio Bulygin's and Cristina Redondo's conceptions about those statements that register the fact that members of a society accept some rules as guides for their behavior. I inquire into what makes it possible for the observer to identify or recognize valid legal rules as such, and my answer is inspired by Wittgenstein's rule-following considerations, taken up from Brandom's critique against "regularism". I concur with Redondo on those points where she is in disagreement with Bulygin, although I express a slight but crucial difference with Redondo's position.

KEY WORDS: internal point of view, descriptive/prescriptive, rule-following, observer, "regularism"

\section{Introducción}

A propósito del trabajo titulado "Los enunciados jurídicos internos. La concepción de Eugenio Bulygin" (Redondo 2013) y de la respectiva respuesta de Bulygin ("Algunas respuestas a los críticos", 2013) se entabla un diálogo entre estos dos eminentes autores. El tema en disputa parece ser ${ }^{1}$ el carácter de los enunciados que Hart (1963, p. 309) distingue — de otros dos tipos de enunciados externos-

${ }^{1}$ Digo "parece" porque lo singular del "diálogo" es que — hasta donde sé- los autores no han logrado ponerse de acuerdo acerca de si estaban o no en desacuerdo. Es difícil determinar si se trata de un malentendido, pero mientras que en su respuesta Bulygin se preguntaba "Entonces ¿en qué estamos en desacuerdo?" (2013, p. 254), supongo que Redondo insistiría en atribuirle las inconsistencias señaladas en su trabajo. Tratar de desentrañar si hay o no un genuino desacuerdo entre ambos y en tal caso en qué punto reside el mismo es parte de lo que me propongo en este trabajo. 
como aquellos realizados por el observador que "no sólo registra meras regularidades y reacciones hostiles, sino también el hecho de que los miembros de la sociedad aceptan ciertas reglas como pautas o criterios de conducta", ${ }^{2}$ y a los que Bulygin (1991a, p. 183) se refiere como "enunciados externos del tercer tipo".

El artículo de Redondo comienza planteando cuestiones relativas a qué tipo de entidades son las normas jurídicas, desestimando la opción planteada por Alchourrón y Bulygin entre una concepción hilética y una expresiva, en la medida en que se entienda como una dicotomía excluyente. Redondo propone una tercera opción, basada en la concepción de Searle sobre las realidades institucionales; continúa sosteniendo que las normas son entidades abstractas, pero cuya existencia depende de hechos empíricos (2013, p. 174). No es gratuito que el trabajo de Redondo comience por allí. Su propuesta — si la comprendí correctamente - es que esos hechos empíricos de los cuales depende la existencia de normas jurídicas (p. ej., la promulgación, la inscripción en un documento, etc.) sólo son generadores de normas jurídicas en cuanto se verifique otro hecho: la aceptación, por parte de un grupo, de un "patrón constitutivo de base" (2013, p. 175).

Redondo usa el término "patrón constitutivo de base" y no "regla de reconocimiento" (en adelante RR) entiendo que para evitar confusiones con la aceptación de índole moral que algunos autores - entre los que Redondo incluye a Bulygin - atribuyen a la regla hartiana. Dado que descarto de plano que la RR hartiana implique algún tipo de aceptación moral, ${ }^{3}$ y como considero que, correctamente comprendida, cumple el mismo papel que el que Redondo asigna a su "patrón constitutivo", creo que ambos términos pueden usarse indistintamente, y así lo haré en este trabajo.

Aclarado lo anterior, las tesis que encuentro controvertidas pueden resumirse del siguiente modo.

Para Redondo:

Rl. Cuando - con un objetivo meramente epistémico- identificamos lo que se debe o está permitido hacer en un sistema jurídico estamos en un sentido presuponiendo o colocándonos en el punto de vista interno respecto de ese patrón constitutivo. (2013, p. 180)

${ }^{2}$ La cita corresponde a la nota de la página 110 de Hart 1963.

${ }^{3}$ La aceptación moral no es necesaria ni siquiera por parte de los agentes que se encuentran alcanzados por las consecuencias normativas del sistema en cuestión. Así lo sostiene expresamente Redondo, con quien coincido. Al respecto, véase la nota 9 infra in fine. 
R2. No podríamos "ver" o identificar las normas existentes o válidas en un sistema jurídico sin usar, y en ese sentido aceptar, el contenido de dicho patrón. (2013, p. 180)

Para Bulygin:

B1. Hay enunciados de validez que son genuinas proposiciones descriptivas (enunciados externos del tercer tipo) y que no presuponen ni expresan aceptación de esa regla ni la de la regla de reconocimiento. Enuncian el hecho de que una determinada regla satisface los criterios de identificación suministrados por la regla de reconocimiento. (1991a, p. 181)

B2. Los enunciados internos (formas disfrazadas de formular una regla) no tienen cabida en una ciencia positivista del derecho. (1991a, p. 182)

2. El desacuerdo es sobre el estatus de los enunciados con los que la ciencia jurídica da cuenta de la existencia de normas

De lo anterior parece que Bulygin y Redondo estarían en desacuerdo nada más y nada menos que acerca de cuál es el carácter de los enunciados mediante los cuales la ciencia (positivista) del derecho da cuenta de la existencia de normas.

Si bien ambos coinciden en que los enunciados epistémicos - emitidos por el teórico - son descriptivos, informativos (no son prescripciones), Redondo los sitúa en el punto de vista interno y Bulygin en el punto de vista externo.

De hecho, Bulygin sostiene explícitamente un paralelismo entre punto de vista externo/enunciados descriptivos y punto de vista interno/ enunciados prescriptivos.

Redondo, en cambio, parece asimilar el punto de vista externo al registro de meras regularidades (los enunciados del primer y del segundo tipos de Hart), y el punto de vista interno, al uso de un patrón constitutivo o RR que puede usarse con dos objetivos: a) para describir, o b) para prescribir, en el sentido de entenderse alcanzado, junto con otros, por las consecuencias prácticas.

En resumen, para Bulygin no se necesita aceptar ningún patrón o regla constitutivos para la mera descripción de normas. El teórico puede identificar desde fuera, sin tener que usar ninguna RR. Redondo critica: los enunciados externos del observador de Bulygin no captan normas, sólo registran hechos externos (2013, p. 177); Bulygin responde: obviamente, las proposiciones normativas versan sobre normas (2013, p. 254); mas, en su opinión, para emitir dichas 
proposiciones descriptivas no se necesita colocarse en el punto de vista interno con quienes usan las normas, ya que, en tal caso, el teórico ya habría pasado a prescribir, tarea que le es ajena. He aquí — si no entendí mal- el núcleo del desacuerdo.

Es difícil decir quién de los dos está más cerca de Hart; pero no es ése mi propósito en este trabajo. Con todo, atendiendo al siguiente parágrafo del "Postcript", creo que ambos lo están, aunque en distintos puntos:

Desde luego, un teórico descriptivo del Derecho no comparte él mismo la aceptación de los participantes, pero puede y debe describirla [...]. Es cierto que, para tal fin, el teórico descriptivo del Derecho debe "comprender" lo que significa adoptar el punto de vista interno y, en este sentido restringido, debe estar en condiciones de ponerse en el lugar de un participante; pero esto no equivale a compartir o suscribir el punto de vista interno del participante ni a renunciar de manera alguna a su posición descriptiva. (Hart 1997, p. 95; las comillas son del original.)

En favor de la tesis de Redondo, parece que Hart aquí estaría aceptando la posibilidad de que el teórico "se coloque" - aunque en un sentido limitado - en el punto de vista interno a fin de "comprender" en qué consiste adoptarlo. En favor de la tesis de Bulygin, Hart señala que ello no implica compartir ese punto de vista interno con los participantes de la práctica ni, por supuesto, aceptar el derecho o dejar de describirlo. Por cierto, Redondo no niega esto último. El punto a dilucidar radicaría más bien en qué quiere decir que el teórico comprenda lo que es adoptar un punto de vista interno sin que ello implique abandonar su posición descriptiva.

\section{Algunas propuestas}

\subsection{Nino: ¿Distintos niveles de punto de vista interno?}

Una propuesta para salir del entuerto sería la señalada por Carlos Nino, en el sentido de que existen distintos niveles de punto de vista interno (1989, p. 41). Quien identifica normas jurídicas válidas con un objetivo meramente descriptivo actuará en un nivel de punto de vista interno (dado por esa identificación en común con quienes usan las normas), pero que no implica el compromiso de someterse a las consecuencias de las mismas.

Con todo, así visto el tema, la salida "a lo Nino" sería una manera algo superficial de solucionar el conflicto, puesto que — si se pone 
entre paréntesis la clasificación "interno" y "externo" - el desacuerdo persiste. Al fin y al cabo la pregunta que debe responderse es ¿qué (si algo) es lo que necesita el teórico "aceptar" o "usar" en común con sus observados para poder comprender/describir las reglas de ese grupo - en el sentido hartiano de guías de conducta- y no meras regularidades? Una posición como la de Redondo responderá que lo que el observador "usa" en común con sus observados es un patrón constitutivo (o RR). La respuesta opuesta - a la manera de Bulygin - responderá que "nada"; el observador puede reconocer, identificar normas en cuanto tales sin usar nada en común con el grupo objeto de estudio. Puede describir algo como fundamento sin tomarlo o aceptarlo como tal.

\subsection{Raz: los enunciados jurídicos no comprometidos}

Por su parte, Joseph Raz $(1982,1999)$ ha propuesto que existe una tercera categoría de enunciados no reducibles ni a los enunciados externos ni a los internos de Hart. Se trata de los "enunciados jurídicos no comprometidos" (detached legal statements). A primera vista podría pensarse que éstos guardan relación con la tesis planteada por Redondo. Pero aquí hay que ir con cuidado porque me parece que lo que plantea Raz — al menos lo que surge de sus ejemplos- incluye tipos diversos de enunciados, no todos asimilables a los de Redondo (que habla exclusivamente del agente que describe la existencia de normas con un objetivo epistémico). En cambio en la categoría de Raz, parecen quedar englobados casos distintos, entre los que pueden identificarse algunas prescripciones. Quien aconseja a otro diciéndole cómo debería actuar está prescribiendo (en un sentido amplio, por supuesto); por ejemplo, el no vegetariano que le dice a su amigo vegetariano "no deberías comer ese plato" (Raz 1999, p. 175); como también el católico experto en tradición rabínica que aconseja a su amigo judío ortodoxo (Raz 1982, p. 198), o el abogado anarquista que aconseja al cliente (Raz 1982, p. 183). ${ }^{4}$ Otro tema es que dicha prescripción sea emitida "como si" se adoptara o "simulando"

${ }^{4}$ A mi modo de ver, el dato de que un abogado sea anarquista resulta totalmente irrelevante para la determinación de que - en la circunstancia en la cual aconseja a su cliente - está actuando desde el punto de vista interno. No sólo está actuando dentro del sistema jurídico en el sentido de que — aunque no le guste- es un sujeto alcanzado por las normas del sistema, sino que, además, está usando dichas normas como guías de conducta en cuanto — más allá de su desacuerdo personal— está intentando dirigir la conducta del cliente para que actúe de acuerdo con las mismas. 
adoptar un punto de vista que, de hecho, no se "acepta". ${ }^{5}$ Pero Raz pone los ejemplos anteriores en la misma categoría de los enunciados emitidos por el teórico (1982, p. 200); y a todos ellos opone, como contrapartida, los enunciados "comprometidos" que para él implican aceptación moral. ${ }^{6}$ Ahora bien, una cosa es aconsejar o responder una pregunta hecha con interés práctico (i.e., "¿qué debo hacer?": la del vegetariano, el judío o el cliente al abogado anarquista) y otra distinta es responder una pregunta teórica con un objetivo meramente epistémico como la que se plantea e intenta responder el teórico, o un profesor de derecho (que es otro de los ejemplos englobados por Raz como enunciados no comprometidos).

Con razón, Bulygin (1991b, pp. 430-431) critica a Raz por no diferenciar adecuadamente los distintos usos del lenguaje - descriptivo y prescriptivo - que están en juego cuando se emiten, respectivamente, enunciados teóricos y prácticos. La misma crítica no es imputable a Redondo, quien restringe su planteamiento específicamente a la labor teórica, a la que caracteriza como descriptiva.

El problema es que Bulygin asume una triple distinción que corre en paralelo: entre enunciados teóricos/descriptivos/externos por un

${ }^{5}$ Kevin Toh (2007) propone esta noción de "simulación" para dar cuenta de la aceptación de un punto de vista en los "enunciados no comprometidos". Pero, a diferencia de Raz, cree que los mismos pueden ser incluidos sin problemas en la categoría de enunciados jurídicos internos, que la teoría de Hart cuenta con los recursos para ello. Toh le critica a Raz que la noción de aceptación desde el punto de vista interno implique aceptación moral; coincido en este punto. Toh plantea, además, otra discusión argumentando que Hart cambió su noción de aceptación plena a una de aceptación débil ("full-acceptance" o "weak-acceptance") con motivo de la crítica de Raz, sin que ello fuera necesario" (Toh 2007, p. 420). No considero necesario dilucidar esa cuestión en el marco de este trabajo. Para lo que aquí planteo me basta con el reconocimiento de que ninguna de las dos nociones de "aceptación" conlleve necesariamente algún elemento moral.

${ }^{6}$ Para dar cuenta del carácter "no comprometido" de los enunciados de la ciencia jurídica, Raz acude al punto de vista del "hombre de derecho"(1982, pp. 183-184): el teórico "adopta" este punto de vista de un modo "especial", de manera "profesional", mientras que la adopción plena o "comprometida" de los individuos es, claramente, aceptación moral ("el individuo que adopta el punto de vista del 'hombre jurídico" considera el derecho como justo"). A diferencia de Raz, Redondo rechaza que el punto de vista interno presuponga aceptación moral, incluso para los participantes de la práctica que se encuentran alcanzados por las normas jurídicas: "Por lo general el patrón de base de un sistema jurídico se acepta por las más diversas razones: por ejemplo, para poder usufructuar los derechos que el sistema confiere, para no perder una fuente de ganancia económica, para no ser castigados. O hasta por ninguna razón: porque sí, por rutina [...]; en este tipo de hipótesis, la aceptación del patrón constitutivo de base no presupone, ni de ella se sigue, una creencia en sus méritos sustanciales, o en su validez absoluta" (Redondo 2013, p. 181). 
lado, y enunciados prácticos/prescriptivos/internos por el otro. La tesis de Redondo constituiría un desafío a cómo la distinción entre el punto de vista interno y el externo juega con los otros dos pares de enunciados al sostener que quien, con un objetivo teórico, describe normas, en algún sentido "acepta" y/o "se coloca" en el punto de vista interno del patrón constitutivo. Se trata de una tesis distinta de la de Raz (que plantea una tercera alternativa a la distinción entre enunciados externos e internos y, además, con sus ejemplos parece establecer relaciones "cruzadas" entre los otros cuatro tipos de enunciados). Por ello, la tesis de Redondo no se hace pasible de las mismas críticas que Bulygin dirige a Raz. ${ }^{7}$

\section{La relación entre los casos de seguimiento y la regla es interna}

Por mi parte, la discrepancia con Bulygin (y coincidencia con Redondo) se apoya en algunos argumentos basados en un enfoque wittgensteiniano. ${ }^{8}$ Siguiendo a Wittgenstein, Robert Brandom (1994,

${ }^{7}$ Otra crítica de Bulygin (1991b) a Raz se basa en que este último pretende comparar los enunciados internos de Hart con las "proposiciones jurídicas" de Kelsen siendo que, para Bulygin, se trata de fenómenos jurídicos distintos. Creo que en este punto contra Raz, Bulygin también tiene razón. Con todo, no estoy segura de que los enunciados no comprometidos de Raz puedan reconstruirse como enunciados condicionales a la manera de "Si se acepta este punto de vista, entonces debe hacerse tal cosa", como propone Bulygin para entonces afirmar que no se trata de enunciados normativos. Véase una crítica a los "enunciados jurídicos no comprometidos" de Raz, pero distinta de la de Bulygin, y que además aborda el tema de cómo la defensa de ese tipo de enunciados se relaciona con las tesis del positivismo jurídico, en Duarte d'Almeida 2011.

${ }^{8}$ En el marco de este trabajo, mi alusión a Wittgenstein se limita al planteamiento acerca del seguimiento de reglas contenido en las Investigaciones filosóficas (§§ 198242 , especialmente el $\S 201$ y el $\S 202$ ) en torno a que seguir reglas constituye una práctica, un know how. Sabido es que el "seguimiento de reglas" ha provocado diversas discusiones exegéticas, y dentro de la teoría del derecho hay diversas líneas en lo relativo a la interpretación de normas jurídicas: están quienes, siguiendo la particular lectura de Kripke (1982), asumen una postura escéptica y, en el otro extremo, quienes poniendo el énfasis en la existencia de acuerdos, como Patterson (1990) o Marmor (2001), parecerían no poder dar cuenta de la discrepancia. No me identifico con ninguna de ellas. Definitivamente, Wittgenstein no es un escéptico; por el contrario, denuncia el malentendido en el que incurre el escéptico; dice de él que ha planteado mal las cosas; pero tampoco me parece que se pueda apelar a sus reflexiones para hablar de algo así como una "gramática del derecho" (Langille 1988), o de un "juego del lenguaje jurídico". En términos generales, leo a Wittgenstein en la línea de David Pears (1988) y también de Brian Bix (1993). Sin embargo, discrepo de este último en que las consideraciones del seguimiento de reglas no tengan relevancia en el ámbito jurídico, o en todo caso tengan una aplicación muy limitada (Bix señala que las reflexiones wittgensteinianas serían útiles a lo sumo para 
pp. 26-30) señala que: dado que cualquier conjunto de acciones exhibe muchas regularidades, para reconocer la existencia misma de una regla y poder considerar una conducta como contraria o acorde a ella, hemos debido ya saber seleccionar algunas regularidades específicas, privilegiadas, de entre todas las exhibidas; ese saber seleccionar — en cuanto puede realizarse de modo correcto o incorrecto- presupone ya estar siguiendo una regla, una práctica normativa (en el caso que nos ocupa, se trataría de la RR). Éste es el argumento que Brandom usa contra lo que denomina "regularismo", el defecto de ver en el comportamiento con base en reglas sólo regularidades de conducta. ${ }^{9}$

dar cuenta de los "casos fáciles"). Discutir aquí este punto me llevaría a abordar cuestiones que exceden ampliamente los límites de este trabajo. Con todo, diré que las consideraciones de Wittgenstein acerca del seguimiento de reglas no constituyen una "teoría" en el sentido de una explicación epistemológica que pretenda ajustarse a un fenómeno que es su objeto; consisten, en cambio, en una elucidación acerca de cómo el lenguaje y nuestras acciones se encuentran interrelacionadas, esto es, acerca de la relación interna que se da entre las reglas y sus aplicaciones. Y en cuanto afirmaciones conceptuales - i.e., no empíricas - atañen a la naturaleza misma de la práctica de seguir reglas, más allá del ámbito (i.e., cotidiano, matemático, jurídico o cualquier otro) de que se trate.

${ }^{9}$ Recogiendo las enseñanzas de Wittgenstein, Brandom (1994, pp. 18-29) se aparta tanto del vicio del "regularismo", como del "regulismo". El primero, en la medida en que sólo atiende a una regularidad coincidente con un patrón, no puede trazar una distinción entre el actuar en virtud de la regla o simplemente caer bajo un patrón de conducta, y pierde así la distinción entre lo que se hace y lo que se debe hacer; i.e., la noción de normatividad. Por su parte, el regulismo asimila lo normativo a las reglas o principios explícitos, considerando que una conducta es apropiada o inapropiada precisamente en cuanto está regida por una regla explícita que así lo dice. Para el inferencialismo de Brandom, el entendimiento, la facultad conceptual, es la facultad de captar reglas, de apreciar la distinción entre la aplicación correcta y la incorrecta que (éstas) determinan. Se trata de una concepción normativa: conocer el significado de las palabras u oraciones implica saber usarlas correctamente. Así, el inferencialismo normativo de Brandom se diferencia de las teorías que explican la competencia lingüística en términos causales, como por ejemplo las herederas de un enfoque chomskiano (Fodor y Lepore 1992) y/o las llamadas semánticas de roles inferenciales que sostienen que el significado consiste en $-\mathrm{o}$ es causado porciertos sucesos: estados mentales, o las inferencias que, de hecho, un individuo realiza (p. ej., Boghossian 1993). En el marco de una concepción normativa, la explicación del significado no puede consistir en señalar una coincidencia (i.e., el hecho de que todos actuamos - individualmente - de cierta manera) o en dar cuenta de una sumatoria de estados psicológicos o de disposiciones individuales; por el contrario, requiere reglas, esto es, criterios intersubjetivos de corrección e incorrección. A fin de puntualizar esta cuestión relativa a la normatividad del significado, traigo aquí el inferencialismo de Brandom. Para una crítica a las teorías lingüísticas "causales", véase Baker y Hacker 1984 (pp. 254-298). Y para una crítica al "inferencialismo causal" desde el punto de vista del "inferencialismo normativo" de Brandom, véase Peregrin 2014 (pp. 8-11). 
Utilizo el ejemplo de Hart (1963, p. 156) del padre que le enseña a su hijo la regla de sacarse el sombrero al entrar a la iglesia: ${ }^{10}$ el hijo que sabe actuar "igual", o hacer "lo mismo" que el padre, ha sabido seleccionar qué aspectos de la conducta del padre son los relevantes; así, ha debido descartar como irrelevantes otras regularidades concomitantes, por ejemplo, que se lo saque con la mano izquierda o con la derecha, lenta o rápidamente, que lo coloque debajo del asiento, etc. Para ello ha usado unos criterios (pese a ser incapaz de especificarlos o de listar todos los aspectos relevantes que ha considerado o los que ha descartado como irrelevantes); y en este sentido, ha seguido la regla. Del mismo modo, ante una multiplicidad de acciones que los agentes observados desarrollan regularmente, el observador, para poder reconocer la existencia misma de la regla, debe utilizar los criterios que aquéllos usan. La idea general se plasma en palabras de Narváez Mora:

La posibilidad [...] de ofrecer una descripción proviene de haber usado ya una regla conceptual, o, si se quiere, de tener en cuenta unos criterios. [...] Sin algún esquema de interpretación no se puede realizar ninguna descripción de los comportamientos que son casos de seguimiento, uso o aplicación de una regla; porque la relación entre los casos de seguimiento y la regla es interna, la regularidad que puede ser vista constituye la aplicación de una regla. (2003, p. 263; las cursivas son mías.)

Es de notar que estos argumentos parecen referirse a reglas primarias. Su transpolación al caso de una regla secundaria como la RR quizás merecería alguna consideración adicional, pero no me parece determinante ocuparme de ello aquí. Lo que me interesa es agregar a las puntualizaciones conceptuales precedentes una propia, tendiente a cuestionar el planteamiento de Bulygin de un paralelismo entre enunciados descriptivos/punto de vista externo y enunciados prescriptivos/punto de vista interno. ${ }^{11}$

${ }^{10} \mathrm{El}$ ejemplo es usado por Hart al comienzo del capítulo VII de El concepto de Derecho para ilustrar el tema de la indeterminación de las reglas en el sistema de precedentes: aquel supuesto en el que, prescindiendo del uso de palabras generales, se ordena seguir una regla mediante el ejemplo (i.e., se debe hacer "lo mismo").

${ }^{11}$ Carlos Nino es uno de los autores que sostienen explícitamente la falta de coincidencia entre la distinción entre el punto de vista interno y el externo y la distinción entre lo normativo y lo descriptivo (1989, p. 39). Sin embargo, en ocasiones su postura parece tender a confundir el punto de vista interno (aunque no específicamente el del derecho, mas sí el de la praxis humana) con algún punto de vista moral (1989, pp. 41-42). 
Decir que se acepta una regla constitutiva (lo que implicaría actuar desde el punto de vista interno de aquello que la regla constituye) y aun así se está describiendo o informando, resulta perfectamente inteligible.

Piénsese en la siguiente analogía: necesito usar/aceptar las reglas constitutivas del castellano y de la lógica para poder emitir enunciados descriptivos; aun para describir las reglas del lenguaje mismo. En ese caso, es perfectamente factible sostener que para poder describir necesito haber aceptado ya determinada práctica normativa (patrón, regla constitutiva, etc.). Y nuestro actuar desde un punto de vista interno respecto del lenguaje no impide trazar luego la diferencia entre describir y prescribir (dentro del lenguaje).

Lo que el ejemplo anterior muestra es que hay un caso, el del lenguaje, en el que el paralelismo entre el punto de vista externo/enunciados descriptivos y el punto de vista interno/enunciados prescriptivos no funciona. Ahora bien, éste podría ser el único caso, debido al carácter universalista, inescapable, del lenguaje; ${ }^{12}$ pero al menos muestra que el paralelismo que sostiene Bulygin no es necesario.

Creo que podría afirmarse que el reconocimiento de normas jurídicas con base en una RR conlleva un elemento de carácter realizativo (en el sentido de los performatives austinianos), esto es, que al decir algo (i.e., emitir un enunciado que dice que algo es una norma válida) se hace otra cosa (en el caso, usar, aceptar la RR). Esta aceptación no presupone deliberación ni ponderación.

Quien actúa desde el punto de vista interno como participante de la práctica difícilmente expresa enunciados de ese tipo. Es más bien al comportarse de determinada manera en razón de las reglas (i.e., al criticar los desvíos de los otros y a su vez considerarse pasible de crítica) cuando - desde el punto de vista externo - decimos que acepta la RR. Su aceptación se muestra en el modo en que participa de la práctica con otros, como sujeto alcanzado por las normas. Pero los participantes no necesitan expresar la RR ni siquiera, quizás, representársela.

En el caso del observador, éste no participa de la práctica comportándose o ajustando su conducta de tal o cual manera en razón de las reglas, puesto que las mismas no lo alcanzan. Sin embargo, con Redondo (y frente a Bulygin) puede decirse que al identificar - descriptivamente - ciertas normas como válidas, éste usa (inevitablemente) el patrón constitutivo o RR; porque el reconocimiento

\footnotetext{
${ }^{12}$ Sobre el tema de la universalidad del lenguaje puede verse Hintikka y Hintikka 1996.
} 
mismo de esos casos como casos de la regla (i.e., la selección de las mismas regularidades como privilegiadas) implica el uso de ésta. Al caracterizar como "válidas" las normas que cumplen con los requisitos establecidos en el patrón constitutivo, el observador ha compartido los mismos criterios identificatorios que el grupo.

La idea clave radica en el carácter práctico y normativo (en contraposición a conceptual) que tiene, a mi modo de ver, el seguimiento de reglas. Respecto de la $R R$, este carácter es tal, ya sea que se trate de la identificación de normas del sistema propio o de uno ajeno, es decir, tanto si las consecuencias normativas del sistema nos alcanzan, como si no. Porque saber seguir una regla — de cualquier tipo, aun si se trata de una regla conceptual o determinativa como Bulygin considera a la RR (sea lo que sea lo que quiere decir con ello) - no es, en sí mismo, una cuestión de "satisfacer definiciones": es una práctica normativa que implica el uso, con otros, de criterios de corrección y la asunción de determinados compromisos.

En el caso del observador, esos "compromisos" (que, reitero, en ningún sentido implican considerarse un sujeto alcanzado por las normas jurídicas identificadas) son los relativos a sus afirmaciones de que tales y cuales son normas válidas. En términos de Brandom (1994), afirmar algo implica la realización de una acción, y esto conlleva adquirir compromisos como el de defender su contenido y sus consecuencias frente al cuestionamiento, aceptar las premisas de las que dicha afirmación es consecuencia y desafiar a quien no los acepte para casos similares. ${ }^{13}$

\section{Seguir reglas es una práctica}

He dicho que coincido con Redondo en lo que ella discrepa con Bulygin (i.e., el uso por parte del observador de la RR para la descripción de las normas jurídicas del grupo observado). Ahora desplazaré el eje del argumento hacia donde, a mi entender, radica la clave de lo que permite al observador hablar de la validez de esas normas o de normatividad jurídica. Sostengo que no es, como Redondo lo propone, el uso mismo de la RR del grupo observado.

${ }^{13}$ Para Brandom, realizar una afirmación implica adquirir determinados compromisos y habilitaciones. En este sentido, la práctica lingüística es en sí misma normativa. Aquí, el término "normativa" tiene una connotación distinta y más amplia que "prescriptiva". De hecho, Brandom otorga primacía al acto de habla aseverativo respecto de otros tipos de usos del lenguaje, dado que, según considera, es el que puede actuar como razón o por el cual se pueden pedir razones; y, para Brandom, la clave de la práctica lingüística radica en este dar y pedir razones entre los miembros de la comunidad. 
Comienzo por introducir dos ejemplos:

Ejemplo 1. Puedo describir las reglas de uso de un idioma extranjero: para ello, por cierto, deberé usarlo (en algún sentido), digamos, para confeccionar mi diccionario o manual de traducción. Pero aún antes de eso, para que el idioma extranjero no constituya para mí meros ruidos, debí haber aprendido a hablar (alguna lengua), debí haber participado como hablante en la práctica de mi lengua materna.

Ejemplo 2. Para que un observador pueda describir las reglas de un juego que no está jugando, tiene que haber participado alguna vez como jugador en algún juego. Sólo ese saber práctico de jugador le permite comprender en qué consiste que algo sea una regla de un juego (i.e., distinta de las reglas sociales, de las de una guerra, u otras) y, por lo tanto, le permite describir como tales las reglas de un juego del cual no está participando. ${ }^{14}$

Si se aceptan los dos ejemplos, creo que pueden trasladarse al caso que nos ocupa. Como señala Redondo, cuando el teórico describe una conducta como prohibida, permitida u obligada en un sistema jurídico determinado, identifica normas jurídicas válidas, esto es, obligaciones, permisiones o prohibiciones emanadas de una autoridad competente (sólo que en el caso del observador, lo que reconoce es que esa conducta está prohibida, permitida u obligada para otros, y no para él).

Ahora bien, para poder hacer esto, debe saber ya lo que significa que una conducta esté jurídicamente permitida, prohibida u obligada por el derecho. Este "saber lo que significa" no es meramente semántico o proposicional (know that); no es que el teórico puede reconocer normas jurídicas porque conoce la definición de los términos "jurídicamente prohibido", "jurídicamente permitido" o "derecho válido". Se trata un saber eminentemente práctico; un saber cómo (know how) de carácter interno, adquirido por haber participado él mismo desde el punto de vista interno de la práctica social de verse alcanzado por normas jurídicas como guías de su propia conducta.

Validez o normatividad jurídicas son nociones internas. En el sentido de que sólo las adquirimos mediante la participación efectiva en

${ }^{14}$ Creo que el ejemplo queda iluminado con lo que dice Wittgenstein en el §204 de las Investigaciones filosóficas: "Puedo tal vez, tal y como están las cosas, inventar un juego que nunca sea jugado por nadie.-_Pero sería también posible esto: la humanidad nunca ha jugado ningún juego; pero una vez alguien inventó un juego - que luego en verdad nunca fue jugado?" La respuesta negativa a esta pregunta pone de manifiesto que la comprensión y el uso de los conceptos presupone llevar adelante acciones con otros, prácticas compartidas. 
una práctica intersubjetiva y normativa (i.e., la práctica del derecho). Tengo aquí en mente aquello a lo que Wittgenstein aludía como un acuerdo no en las definiciones, sino en los juicios, en la forma de vida:

$\S 241$. [...] los hombres concuerdan en el lenguaje. Ésta no es una concordancia de opiniones, sino de forma de vida.

$\S 242$. A la comprensión por medio del lenguaje pertenece no sólo una concordancia en las definiciones, sino también (por extraño que esto pueda sonar) una concordancia en los juicios.

Si esto es así, la propuesta de Redondo resultaría insuficiente para explicar cómo es que el observador, sin compartir la práctica efectiva de guiar su conducta por las mismas normas que los del grupo, puede "ver" validez en las normas identificadas en virtud de la RR de ellos. Lo que sugiero es que el hecho de compartir con el grupo observado los criterios identificatorios determinados por la $\mathrm{RR}$ respectiva (i.e., de usar o "aceptar" con ellos esa RR) no basta, por sí solo, para dar con la noción de normas válidas.

Mi idea es que sólo porque en la forma de vida del observador existe el fenómeno (artificial, humano, contingente) de la normatividad jurídica — en el cual él ha participado actuando como sujeto alcanzado por las normas (y no como observador) - puede entonces reconocer, aun en un sistema distinto del propio, que hay hechos externos cualesquiera que dan lugar a ella. ${ }^{15}$

Piénsese en el siguiente ejemplo: nosotros podemos reconocer que en un califato lo que es derecho deriva de los dichos de un califa e identificar las normas jurídicas de ese régimen, aunque se trate de un criterio identificatorio de validez jurídica muy distinto del que poseemos. La asunción de que los dichos del califa están dotados de normatividad para ellos conlleva algún tipo de "acuerdo en los juicios" con ellos. ¿Qué tipo de acuerdo? Por cierto, aquel al que refiere Redondo que implica la identificación de algo en cuanto algo (i.e., los dichos del califa en cuanto normas jurídicas); éste es un reconocimiento, criterio o regla compartidos por el observador y sus observados que están usando — aunque con distintos objetivos- la

${ }^{15}$ Por supuesto, hay un sentido en que esto resulta trivial, porque en las sociedades modernas todo teórico del derecho se encuentra, de hecho, inmerso en algún orden jurídico en el que actúa desde el punto de vista interno, asumiéndose como sujeto alcanzado por las consecuencias prácticas que se siguen del mismo. De allí que parezca una verdad indiscutible que ningún teórico del derecho tiene un punto de vista externo extremo. 
RR del sistema en cuestión. Pero también, y como condición de lo anterior, una suerte de "consenso práctico" que hemos incorporado participando de la práctica del derecho, respecto de qué es derecho válido o qué es que algo esté jurídicamente obligado, prohibido, etcétera.

Así, lo que permite al observador "ver" normas jurídicas (en un grupo a cuyas normas no se encuentra sometido), esto es, lo que necesita para comprender que determinados actos constituyen el fundamento de validez de ciertas reglas y no meros hechos externos, no es, como Redondo sostiene, el uso de la RR del sistema observado: es el saber práctico adquirido únicamente por haber participado él mismo desde el punto de vista interno - en sentido fuerte de estar sujeto a consecuencias normativas - de algún (su) sistema jurídico. ${ }^{16}$

Por ello, coincido con lo que Redondo señala a Bulygin de que no podríamos reconocer la validez de normas jurídicas ajenas sin usar la RR de ese sistema; pero a su vez señalo a Redondo que no podríamos usar con fines meramente descriptivos una RR ajena sin haber sido antes participantes de la práctica normativa interna de algún (nuestro) sistema jurídico. Sólo puede uno "colocarse" o "presuponer" el punto de vista interno del sistema que describe porque alguna vez ha actuado desde el punto de vista interno participando normativamente de la práctica del derecho en el marco de algún sistema jurídico.

\footnotetext{
${ }^{16}$ Con posterioridad a una versión previa de este trabajo llegó a mi conocimiento un trabajo de Bruno Celano (2016), en el que el autor introduce la noción de "preconvenciones": prácticas incorporadas (embodied) no respaldadas por un curso de razonamiento; comportamientos convergentes que no son asimilables a una mera regularidad — porque son normativos - , pero que en algún sentido son automáticos, irreflexivos, effortless, es decir, una suerte de know how irreductible a un know that. Se diferencian así de las "convenciones" que — si bien tácitas o expresasconstituyen acuerdos si respaldados por una estructura de raciocinio (backed by a train of reasoning) como, por ejemplo, las convenciones entendidas à la Lewis como soluciones a problemas de coordinación. Celano acude a las nociones de trasfondo o background (Searle) y "forma de vida" (Wittgenstein) para afirmar que las mismas albergan preconvenciones, y sostiene además que nuestra competencia conceptual está entretejida con preconvenciones (2016, p. 42). Encuentro esta categoría bastante útil para lo que he querido expresar aquí. Utilizándola para mi fines (Celano no toca aquí el tema jurídico) se podría decir que un agente que actúa con otros como participante desde el punto de vista interno de su propio sistema jurídico "incorpora" una preconvención acerca de lo que para él constituye la idea de "derecho válido"; luego, en cuanto observador de un grupo distinto, usa la RR de ese grupo - que para él será una convención-. Pero sólo porque tiene incorporada esa preconvención acerca del derecho puede "ver" o identificar, usando la convención de otros, normas válidas. Para los observados, entre tanto, su propia RR tendría el carácter de preconvención. De todos modos, ésta es una idea tentativa; el uso de estas categorías con relación a la RR requiere una elaboración y un debate mayores de los que puedo abordar aquí.
} 


\section{Conclusiones}

Para finalizar, resumo las cuestiones controvertidas aquí planteadas: i) la que encuentro como discrepancia fundamental entre Bulygin y Redondo: si el observador usa la RR, lo que conlleva "colocarse en" el punto de vista interno; ii) la que he propuesto aquí acerca de qué necesita el científico tener en común con un grupo para poder comprender/describir las reglas del mismo (si acaso hay algo que necesite), y iii) la que se deriva del análisis de Redondo de qué es lo que posibilita al observador "identificar", "reconocer" normas jurídicas válidas en cuanto tales.

En lo que respecta a mis conclusiones, por un lado, y a partir de los argumentos aquí vertidos, discrepo de Bulygin que responde "no" y "nada" a las cuestiones $i$ y ii. Por el otro, coincido con Redondo en $i$ : que el teórico que identifica las normas de un sistema usa la RR del sistema que describe, y entonces actúa - aunque con un objetivo descriptivo diferente del de los participantes - desde el punto de vista interno. Pero destaco entonces cuál sería mi sutil -aunque crucial - diferencia con Redondo respecto de $i i$ y iii: su propuesta es que es el uso de la RR del sistema observado lo que permite al observador reconocer esas normas jurídicas en cuanto tales. En cambio, he puesto el énfasis en que no es (o al menos no es sólo) el uso de esa RR lo que le permite "ver" allí normas jurídicas o atribuir a ciertos hechos externos la propiedad de constituir fundamento de validez. Lo que hace posible al observador identificar normas jurídicas que no lo alcanzan con base en una RR ajena, es el punto de vista interno de $s u$ sistema jurídico, adquirido por su participación en una práctica social o forma de vida en la que la RR de su sistema funciona como fundamento de normatividad. ${ }^{17}$

\section{BIBLIOGRAFÍA}

Alchourrón, C.E. y E. Bulygin, 1991, Análisis lógico y Derecho, Centro de Estudios Constitucionales, Madrid.

${ }^{17}$ Una versión preliminar de este trabajo fue presentada en el taller "Bulygin's Philosophy of Law", celebrado en el marco del XXVII Congreso Mundial de la IVR Washington 2015. Agradezco a quienes leyeron y realizaron comentarios a una primera versión, entre ellos Cristina Redondo, Eugenio Bulygin, José Juan Moreso, Martín Böhmer, y mis compañeros del Seminario de Lógica y Teoría del Derecho de la Facultad de Derecho de la Universidad de Buenos Aires, dirigido por Eugenio Bulygin. Agradezco, asimismo, al evaluador anónimo por sus comentarios, los cuales me han permitido introducir mejoras y aclaraciones al texto original. 
Baker, G. y P. Hacker, 1984, Language, Sense and Nonsense, Basil Blackwell, Oxford.

Bix, B., 1993, Law, Language, and Legal Determinacy, Clarendon Press, Oxford.

Boghossian, P., 1993, "Does an Inferential Role Semantics Rests Upon a Mistake?", Mind and Language, vol. 8, no. 1, pp. 27-40.

Brandom, R., 1994, Making It Explicit. Reasoning, Representing and Discoursing Commitment, Harvard University Press, Cambridge Mass.

Bulygin, E., 2013, "Algunas respuestas a los críticos", Análisis Filosófico (Homenaje a Eugenio Bulygin), vol. 33, no. 2, pp. 249-264.

— , 1991a, "Normas, proposiciones normativas y enunciados jurídicos", en Alchourrón y Bulygin 1991, pp. 169-193. [Versión original en inglés: "Norms, Normative Propositions, and Legal Statements", Contemporary Philosophy. A New Survey, vol. 3, 1982, pp. 127-152.]

—_, 1991b, "Enunciados jurídicos y positivismo: respuesta a Raz", en Alchourrón y Bulygin 1991, pp. 427-438. (Publicado originalmente en Análisis Filosófico, vol. 1, 1981.)

Celano, B., 2016, "Preconvenciones: un fragmento del trasfondo", en L. Ramírez Ludueña y J. Vilajosana (eds.), Convencionalismo y Derecho, Marcial Pons, Madrid/Barcelona, pp. 25-51.

Duarte d'Almeida, L., 2011, "Legal Statements and Normative Language", Law and Philosophy, vol. 30, pp. 167-199.

Fodor, J. y E. Lepore, 1992, Holism: a Shopper Guide, Blackwell, Oxford. Hart, H.L.A., 1997, "Postscriptum", La decisión judicial. El debate HartDworkin, trad. Magdalena Holguín, Universidad de los Andes/Siglo del Hombre, Bogotá, pp. 89-141. , 1994, The Concept of Law, 2a. ed., Clarendon Press, Oxford.

— 1963, El concepto de Derecho, trad. Genaro R. Carrió, Abeledo Perrot, Buenos Aires.

Hintikka, J. y M. Hintikka, 1996, "Wittgenstein and Language as the Universal Medium", en J. Hintikka, Lingua Universalis vs. Calculus Ratiocinator: an Ultimate Presupposition of Twentieth-Century Philosophy, Kluwer Academic Publishers, Dordrecht (Jaako Hintikka Selected Papers, 2), pp. 163-189.

Kripke, S., 1982, Wittgenstein a propósito de reglas y lenguaje privado, trad. J. Rodríguez Marqueze, Tecnos, Madrid.

Langille, B., 1988, "Revolution without Foundation: The Grammar of Scepticism and Law", McGill Law Journal, vol. 33, no. 3, pp. 451505.

Marmor, A., 2001, "Exclusive Legal Positivism", Positive Law and Objective Values, Oxford University Press, Oxford, pp. 47-70.

Narváez Mora, M., 2003, Wittgenstein y la teoría del derecho, Marcial Pons, Madrid/Barcelona.

Nino, C., 1989, El constructivismo ético, Centro de Estudios Constitucionales, Madrid. 
Patterson, D., 1990, "Law's Pragmatism: Law as Practice and Narrative", Virginia Law Review, vol. 76, no. 5, pp. 937-996.

Pears, D., 1988, The False Prison. A Study of the Development of Wittgenstein's Philosophy, vol. 2, Oxford University Press, Nueva York. Peregrin, J., 2014, Inferentialism. Why Rules Matter, Palgrave Macmillan, Londres.

Raz, J., 1999, Practical Reasons and Norms, Oxford University Press, Oxford/Nueva York.

—_ 1982, La autoridad del Derecho. Ensayos sobre Derecho y moral, trad. Rolando Tamayo, Universidad Nacional Autónoma de México, México.

Redondo, M.C., 2013, “Los enunciados jurídicos internos. La concepción de Eugenio Bulygin", Análisis Filosófico (Homenaje a Eugenio Bulygin), vol. 33, no. 2, pp. 170-185.

Toh, K., 2007, "Raz, on Detachment, Acceptance and Describability", Oxford Journal of Legal Studies, vol. 27, no. 3, pp. 403-427.

Wittgenstein, L., 1988, Investigaciones filosóficas, trad. A. García Suárez y U. Moulines, Crítica/Instituto de Investigaciones Filosóficas-UNAM, Barcelona/México.

Recibido el 21 de septiembre de 2015; revisado el 11 de julio de 2016; aceptado el 3 de febrero de 2017. 